\title{
CLOUD REMOVAL FROM SENTINEL-2 IMAGE TIME SERIES THROUGH SPARSE RECONSTRUCTION FROM RANDOM SAMPLES
}

\author{
D. Cerra*, J. Bieniarz, R. Müller, P. Reinartz \\ German Aerospace Center (DLR), Earth Observation Center (EOC), 82234 Weling, Germany \\ (daniele.cerra, jakub.bieniarz, rupert.mueller, peter.reinartz)@dlr.de
}

Commission III, WG III/3

KEY WORDS: Sentinel-2, Clouds, Image Time Series, Image processing, Pre-processing.

\begin{abstract}
:
In this paper we propose a cloud removal algorithm for scenes within a Sentinel-2 satellite image time series based on synthetisation of the affected areas via sparse reconstruction. For this purpose, a clouds and clouds shadow mask must be given. With respect to previous works, the process has an increased automation degree. Several dictionaries, on the basis of which the data are reconstructed, are selected randomly from cloud-free areas around the cloud, and for each pixel the dictionary yielding the smallest reconstruction error in non-corrupted images is chosen for the restoration. The values below a cloudy area are therefore estimated by observing the spectral evolution in time of the non-corrupted pixels around it. The proposed restoration algorithm is fast and efficient, requires minimal supervision and yield results with low overall radiometric and spectral distortions.
\end{abstract}

\section{INTRODUCTION}

Satellite Image Time Series (SITS) are sets of images acquired by the same or different spaceborne sensors over the same area at multiple acquisition times. In recent years, both the availability and the temporal resolution of SITS acquired by multispectral sensors is increasing steadily. An important challenge for the future is the processing of SITS acquired by the Sentinel-2 mission, which will make available multispectral SITS at global scale with a frequent revisit time of less than 5 days, once both satellites of the constellation will be in orbit (Drusch et al., 2012). It would be desirable then to identify algorithms requiring limited supervision and computational resources to improve the automated processing chain of these datasets.

One hindrance for the analysis of SITS acquired by optical sensors is the presence of thick clouds in the scenes within a multitemporal stack, which makes difficult to observe the evolution of a given ground cover in time. In recent years, researchers have found sparse reconstruction techniques to outperform traditional methods based on based on temporal replacement or patch-based spatial replacement (Shen et al., 2015).

Recently, Sparse Unmixing-based Denoising (SUBD) has been successfully applied for the inpainting of missing values in optical earth observation data. SUBD estimates the value of corrupted pixels based on their sparse decomposition in terms of uncorrupted image elements, making only an indirect use of spatial information. In the case of scenes affected by thick clouds within a SITS, an effective restoration is usually possible if information about the missing image elements is given in cloud-free acquisitions in the multitemporal stack. A large set of cloud-free pixels can be selected to model the evolution in time of the ground covers in a given scene, as sparse methods excel at handling what are known as overcomplete dictionaries, i.e. a set of training samples which can be much larger than the dimensionality of a dataset, which for a multispectral SITS is proportional to the number of spectral bands per image and to the number of temporal acquisitions.

${ }^{*}$ Corresponding author
The efficiency and automation of SUBD can increase when the atoms in the overcomplete dictionary used for the reconstruction are randomly selected from the image, as selecting a large number of elements in the dictionary increases the performance minimising the reconstruction error yielded by a given dictionary. This allows providing results in quasi real-time. Furthermore, the selection of a random dictionary implies that dictionary selection, usually requiring several parameters to set and computational resources when carried out by explicitly avoiding coherent entries or minimising reconstruction errors, only requires the setting of the number of elements to be extracted. This can be chosen according to the size of the analysed images and the number of cloud-free acquisitions available for a given stack. Another advantage of working with sparse methods is their efficiency, as it is possible to use specialized algorithms and data structures exploiting the sparse nature of the handled data, requiring limited computational resources with respect to methods which operate on dense matrix structures.

This paper presents the first results of applying SUBD for sparse reconstruction of missing information in multitemporal Sentinel2 datasets, based on non-corrupted samples randomly acquired from the same images. A mask for clouds and clouds shadows must be given, and it is manually derived in our case. Given the difficulties in the future to process the large amount of Sentinel-2 multitemporal data stacks that will be available, this paper also moves towards an automation of the method, indicating how to set adaptive thresholds in order to process these datasets in an unsupervised way.

The paper is structured as follows. Section 2 gives a reminder on the sparse reconstruction techniques adopted by the algorithm. Section 3 reports the first experimental results of the method applied to a Sentinel-2 data stack. We conclude in Section 4.

\section{SPARSE UNMIXING-BASED DENOISING (SUBD)}

Sparse Unmixing-based Denoising (SUBD) has been defined in (Cerra et al., 2016) and used to reconstruct images contaminated by thick clouds in image time series. The main idea at the basis of the cloud removal method presented therein goes back to 
Unmixing-based Denoising (UBD), an algorithm which in its orig inal conception restored bands affected by a low Signal-to-Noise Ratio (SNR) in hyperspectral images (Cerra et al., 2014). Hyperspectral datasets feature a high number of narrow, adjacent spectral bands often in the order of hundreds, while as a comparison typical multispectral sensors have less than 10 (broader) bands and Sentinel-2 has 13 bands at different spatial resolutions (Drusch et al., 2012).

UBD is based on the concept of spectral unmixing, which aims at decomposing each hyperspectral image element into a linear (or less often non-linear) combination of signals representing the backscattered solar radiation in each spectral band from a target within the image or analysed in laboratory. The considered targets are typically composed of a single pure material or a homogeneous mixture of materials, and are often called endmembers (Bioucas-Dias et al., 2012): in this paper we simply refer to them as reference spectra in order to adopt a consistent nomenclature. The output of a spectral unmixing process is a set of abundances maps, quantifying the contribution of each reference spectrum to a given pixel. In a linear spectral mixture a pixel $m$ could be expressed as:

$$
m=\sum_{i=1}^{k} x_{i} s_{i}+r
$$

where $x_{1} \ldots x_{k}$ and $s_{1} \ldots s_{k}$ are the fractional abundances for the $k$ available and pre-selected reference spectra, while $r$ is a residual vector containing the portion of the signal which cannot be represented in terms of the basis vectors of choice.

The output of the spectral unmixing process in eq. 1 is then inferred into UBD's reconstruction process. By considering the physical properties of a mixed spectrum, the residual vector $r$ is assumed to be mostly composed by noise and more relevant in spectral bands where atmospheric absorption effects are stronger, and therefore ignored in the reconstruction.

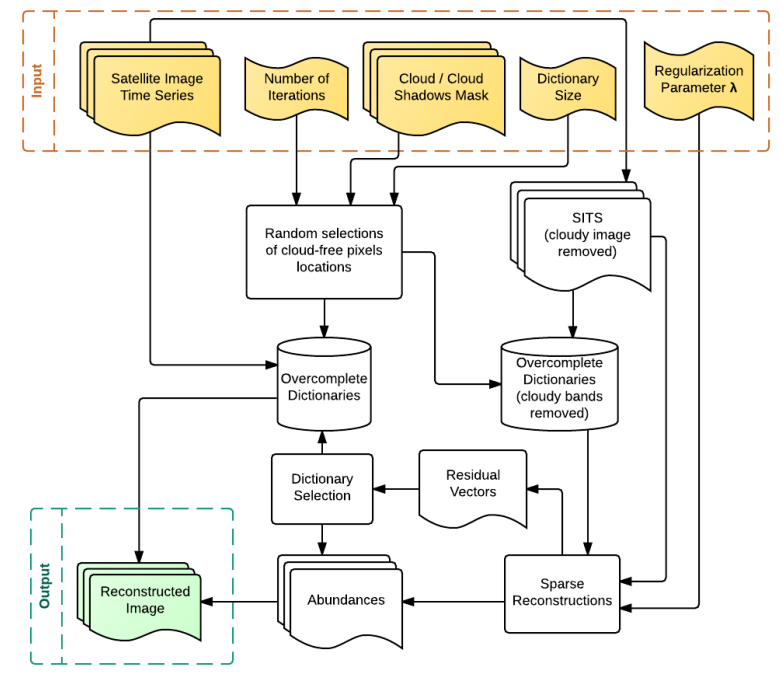

Figure 1. Workflow for the described method. In orange the required input (data and parameters), in green the output. Most of the required input parameters can be empirically or adaptively derived without requiring too much supervision.

Better results have been obtained by coupling UBD with sparse reconstruction techniques (Cerra et al., 2015). In sparsity-based spectral unmixing, most of the abundances $x_{i}$ in Equation (1) are equal to zero, as these methods assume that only a few reference spectra contribute to $m$, and the abundance vector to be estimated is therefore sparse. The use of sparse unmixing within the UBD workflow resulted in the definition of Sparse Unmixingbased Denoising (SUBD), which can be carried out as follows.

In the first step a spectral dictionary $A$ is derived by collecting a large number of image elements, randomly selected from the area which is cloud-free in all the images in the stack. The dictionary is overcomplete, meaning that the number of entries it contains is higher than the dimensionality of the data. The use of overcomplete libraries for sparse reconstruction have been widely used in the past; see, for example, (Iordache et al., 2011), (Bieniarz et al., 2015), and (Tang et al., 2014). Afterwards, each image element $y$ and the dictionary $A$ are fed to a non-negative version of the least angle regression LASSO (LARS/LASSO) reconstruction algorithm (Efron et al., 2004), which guarantees a sparse solution by solving the following minimization problem:

$$
\min _{x}|A \check{x}-\check{y}|_{2}^{2} \quad \text { s.t. } \quad\left|\check{x}_{1}\right| \leq \lambda, \check{x} \geq 0
$$

where $\check{y}$ is the original image from which the corrupted bands have been removed, and $\check{x}$ contains the fractional abundances for the spectra selected in the reconstruction of $\check{y}$, without considering the bands belonging to the corrupted image. The regularization parameter $\lambda$ is the upper bound on the $\ell_{1}$ norm controlling the sparsity of the solution vector $\check{x}$. The regularization problem in eq. 2 is especially advantageous when the dictionary $A$ is overcomplete: this is the case for the large spectral libraries used in SUBD, which are also highly coherent (Bioucas-Dias et al., 2012, Bieniarz et al., 2015). This motivates the choice of the LARS solver, which is robust in dealing with dictionaries having the mentioned characteristics (Bach et al., 2011).

It is important to remark that in the problem $\check{x}$ must be used instead of $x$, as including in the dictionaries the values of corrupted bands (covered by clouds) would introduce relevant error in the abundance estimation step. Instead, the reconstruction is estimated in the cloud-free portion of the stack. To give an informal example, consider a pixel $p_{1}$ below a cloud in the first image of a stack containing $n$ acquisitions, and that in the rest of the stack the spectro-temporal pattern $p_{2 \ldots n}$ is composed by $50 \%$ of $D_{1}$ and $50 \%$ of $D_{2}$, where $D_{i}$ is the $i_{t h}$ entry in a dictionary. If in eq. 2 the bands from $p_{1}$ would be included in the dictionary, the real abundance values could be reconstructed with relevant errors. Instead, the pixel $p_{1}$ can be reliably reconstructed as a subset of $p=0.5 D_{1}+0.5 D_{2}$, as the relative dictionary entries have been collected from areas of the image which are cloud-free also in the corrupted portion of the stack.

With respect to previous works, instead of selecting the median value for each reconstruction after running the process several times, in this paper we select separately the best dictionary for each pixel, chosen in order to yield the minimum value for $r$ after the reconstruction in eq. 1 . This increases the probability that all type of materials or ground covers below the cloud can be correctly reconstructed if they are present also outside of the clouds and selected in any of the dictionaries, as this would yield the minimum reconstruction error.

The full workflow for the method is reported in Fig. 1, in which the required inputs are highlighted. These can be empirically or automatically derived as follows. Firstly, a clouds and cloud shadows mask is easier to automatically retrieve from a SITS than from a single image from dedicated algorithms. Regarding 


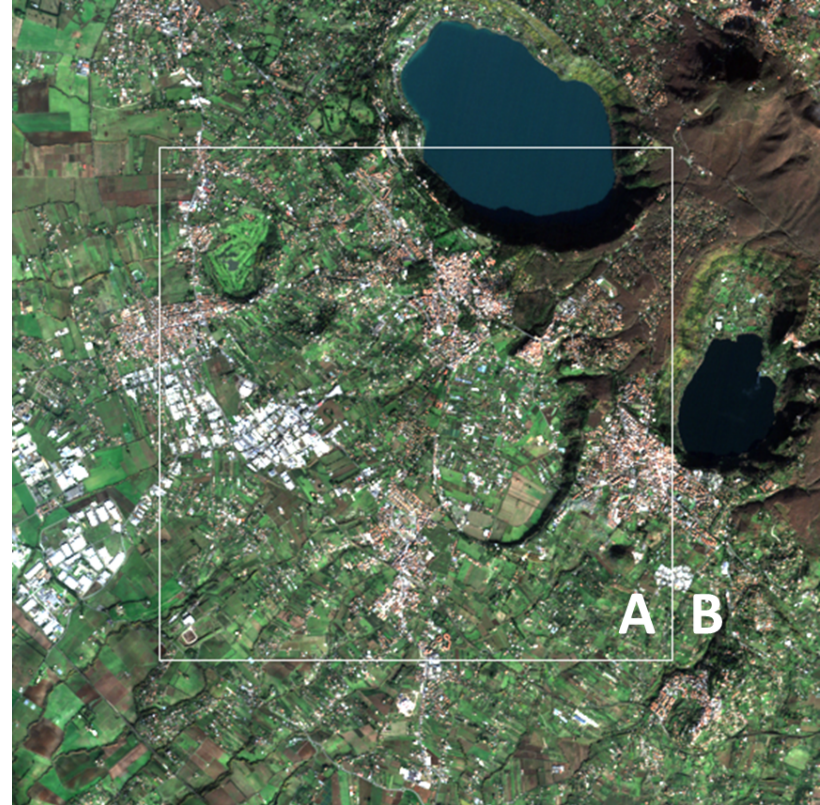

Figure 2. True color combination for a cloud-free subset from the Sentinel-2 Stack analysed in this paper. The area denoted with an $\mathrm{A}$ is assumed to be covered by thick clouds and therefore invisible. The dictionaries used for the sparse reconstruction are built by collecting random pixels from the area in a buffer around the cloud, denoted with a B.

the setting of the $\lambda$ regularization parameter, a value of 1 usually yields satisfactory results whenever the dictionary entries are collected from the same image (Cerra et al., 2016). With the dictionary size empirically set as will be detailed in next Section, the only parameter requiring further investigations is the number of iterations. From preliminary analysis, results did not change significantly after 50 iterations, so we set this specific parameter to 50 in the reported experiments.

\section{EXPERIMENTAL RESULTS}

We analyse a stack of four images acquired over an area close to the city of Rome, Italy, in a timespan ranging from the 18th of December 2015 to the 17 th of January 2016. A $550 \times 550$ subset which is cloud-free in all the images is selected, and only the bands with a spatial resolution of 10 or 20 meters are kept for a total of 10 spectral bands per image. The three bands with a spatial resolution of 60 meters are discarded, and the bands at higher resolution are resampled to 20 meters after applying a Gaussian filter separately to each one to prevent aliasing. Please note that this is done only to simplify the workflow: the bands could be kept at their original resolution by applying the method separately to the three groups of spectral bands, with no degradations in performance (Cerra et al., 2016).

After the subset is selected, occlusion by a cloud of size $350 \times 350$ is simulated in the center of the image (area A in Fig. 2). A total of 50 dictionaries $\mathrm{D}$ is built by collecting for each of those 20 random spectra from the stack in the area outside of the cloud, denoted by a B in Fig. 2. The number of elements $|D|$ for each dictionary $D$ is chosen applying the empirical rule defined in (Cerra et al., 2016):

$$
|D|=\min \{5 N, 100\}
$$

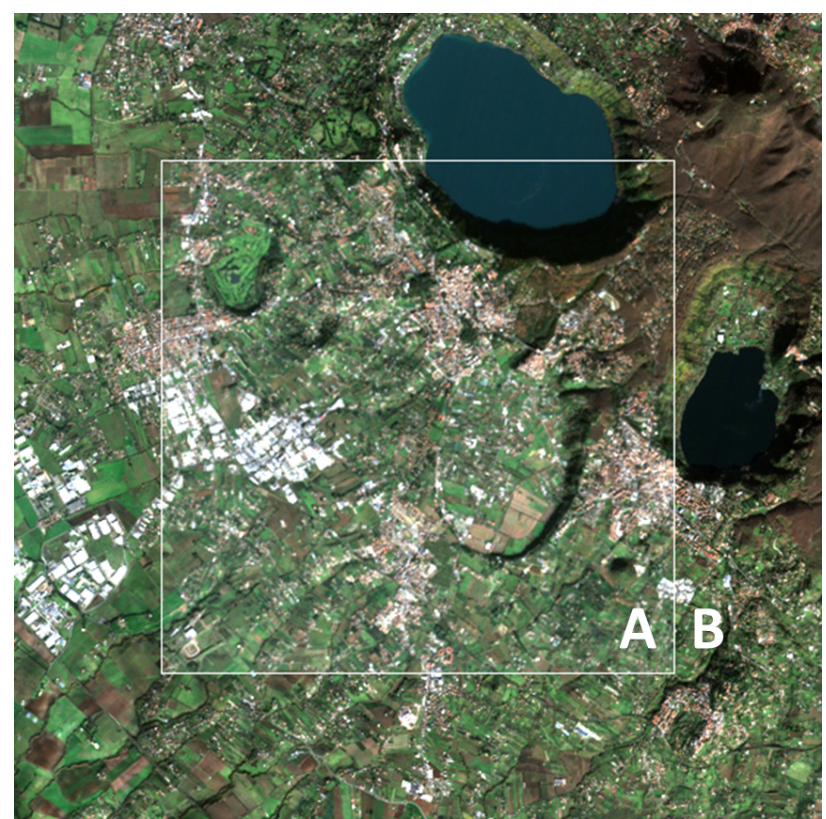

Figure 3. True color combination for the restoration of the area denoted with an A in Fig. 2.

In which $N$ is the dimensionality of the subspace containing the relevant information in the dataset. To estimate $N$ methods traditionally applied to hyperspectral image processing could be used (Bioucas-Dias and Nascimento, 2008). In this case we applied a Principal Component (PC) transform to the Sentinel SITS and select a number of dimensions which contains more than $98.5 \%$ of the total variance of the dataset. For the described stack, these conditions are met by selecting the first four PCs after the rotation, so $N$ is set to 4 and $|D|$ to 20 .

Afterwards, SUBD as described in eq. 2 is applied, and the image is reconstructed according to the dictionary yielding the smallest residual vector for each pixel. Results are reported in Fig. 3. The images are visually very similar, with smooth transitions on the borders between the reconstructed obscured pixels and the cloud-free ones. Results are assessed quantitatively by estimating the absolute reconstruction error and spectral distortion across the 10 considered bands in the affected image. For the former, the figure of merit is the Mean Average Error (MAE) across all the considered 10 spectral bands expressed in percentage, while the latter is quantified through the mean Spectral Angle (SA) (Kruse and al., 1993). Results are reported in Table 1.

\begin{tabular}{|c|c|}
\hline MAE & SA \\
\hline $1.08 \%$ & $4.5 \times 10^{-2}$ \\
\hline
\end{tabular}

Table 1. Mean Average Error (MAE) and Spectral Angle (SA) for the cloud removal experiment in the area denoted by an A in Figs. 2 and 3.

It is possible to spot in the lower part of the larger lake of the reconstructed image a pattern in the water which is not present in the original image. This is due to the fact that, even though the method can succeed at keeping local spectral information, a consistency is assumed between spatial patterns in the rest of the stack. To explain what this could mean in practice, assume having an agricultural field which is partially covered by thick clouds in one image and that its spectrum changes abruptly only in this particular image. If the dictionary used for its sparse reconstruction contains a sample image element from the cloud-free portion 
of that field, this can be reliably reconstructed. On the other hand, local changes on the field below the cloud such as specific crop marks cannot be restored.

Results could be provided to the end users simply as restored images, or by overlaying a transparent white layer on the reconstructed areas, in order to clearly show which pixels have been synthesized and are therefore less reliable than the ones which were cloud-free in the first place. An example for this visualization is reported in Fig. 4.

\section{CONCLUSIONS}

This paper presents the first results of applying Sparse Unmixingbased Denosing (SUBD) to cloud removal in single scenes of Sentinel-2 SITS. Pixels covered by clouds are restored through sparse reconstruction, with the employed dictionaries composed of randomly selected cloud-free pixels.

To increase the automation of the method, a set of dictionary composed by randomly selected image elements is considered, and for the restoration of each pixel the dictionary yielding the minimum reconstruction error in the available cloud-free bands is selected. This allows selecting with high probability a given dictionary which contains the spectro-temporal patterns of the materials of which the pixel of interest is composed. These will be more than one if the image element to be reconstructed is mixed, thing which is likely at Sentinel-2 ground sampling distances. First results on a data stack acquired close to the city of Rome in Italy are satisfactory both objectively and subjectively, as they are visually very similar to the test image and are restored with low reconstruction errors.

The method is not suitable for cases in which information on the contaminated pixels is corrupted but available, as in the case of haze, as it completely ignores the cloudy pixels in the reconstruction process. For this purpose, algorithm coming from the field of atmospheric correction should be employed instead.

In the future, traditional methods based on substitution of the contaminated pixels with cloud-covered ones will be integrated in the workflow. The reconstruction process will resort to these alternative algorithms whenever the residual vector is too high, indicating that probably the materials composing a given pixel below the cloud have not been found in any dictionary extracted from the cloud-free areas around the sensitive one. Another improvement would be represented by a better characterization of the area from which the dictionaries are extracted, increasing the probability that a selected dictionary entry contains a material which is also present to some degree in the area below the cloud. If the cloud mask is available, a possible choice would be creating a buffer around the cloud, and forcing the dictionary entries to be selected from the area defined by the buffer: this would go in the direction of the experiments in Section 3 for the simulated square cloud therein.

\section{REFERENCES}

Bach, F., Jenatton, R., Mairal, J., Obozinski, G. et al., 2011. Convex optimization with sparsity-inducing norms. Optimization for Machine Learning pp. 19-53.

Bieniarz, J., Aguilera, E., Zhu, X. X., Müller, R. and Reinartz, P., 2015. Joint sparsity model for multilook hyperspectral image unmixing. Geoscience and Remote Sensing Letters, IEEE 12(4), pp. 696-700.

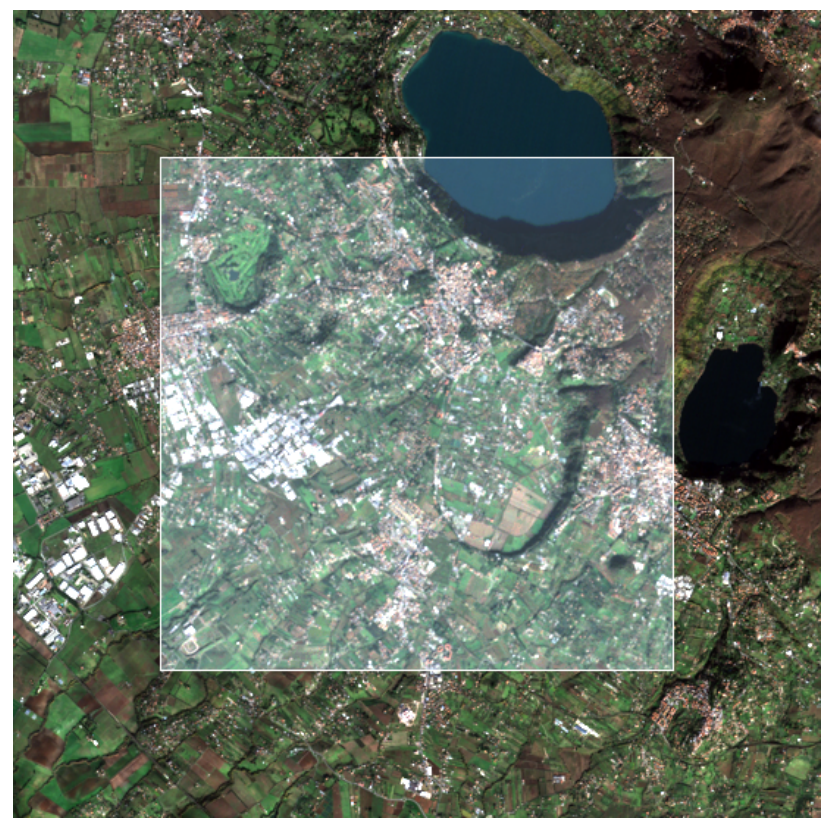

Figure 4. Alternative visualization option for an end user, in which a transparent cloud is simulated and overlaid to visually distinguish original image elements from synthesized ones.

Bioucas-Dias, J. and Nascimento, J., 2008. Hyperspectral subspace identification. IEEE Transactions on Geoscience and Remote Sensing 46(8), pp. 2435 -2445.

Bioucas-Dias, J. M., Plaza, A., Dobigeon, N., Parente, M., Du, Q., Gader, P. and Chanussot, J., 2012. Hyperspectral unmixing overview: Geometrical, statistical, and sparse regression-based approaches. IEEE Journal of Selected Topics in Applied Earth Observations and Remote Sensing 5(2), pp. 354-379.

Cerra, D., Bieniarz, J., Beyer, F., Tian, J., Müller, R., Jarmer, T. and Reinartz, P., 2016. Cloud removal in image time series through sparse reconstruction from random measurements. IEEE Journal of Selected Topics in Applied Earth Observations and Remote Sensing.

Cerra, D., Bieniarz, J., Müller, R., Storch, T. and Reinartz, P., 2015. Restoration of simulated enmap data through sparse spectral unmixing. Remote Sensing 7(10), pp. 13190.

Cerra, D., Müller, R. and Reinartz, P., 2014. Noise reduction in hyperspectral images through spectral unmixing. IEEE Geoscience and Remote Sensing Letters 11(1), pp. 109-113.

Drusch, M., Del Bello, U., Carlier, S., Colin, O., Fernandez, V., Gascon, F., Hoersch, B., Isola, C., Laberinti, P., Martimort, P. et al., 2012. Sentinel-2: Esa's optical high-resolution mission for gmes operational services. Remote Sensing of Environment 120, pp. 25-36.

Efron, B., Hastie, T., Johnstone, I. and Tibshirani, R., 2004. Least angle regression. Annals of statistics 32(2), pp. 407-499.

Iordache, D. M., Bioucas-Dias, J. and Plaza, A., 2011. Sparse unmixing of hyperspectral data. Geoscience and Remote Sensing, IEEE Transactions on 49(6), pp. 2014-2039.

Kruse, F. and al., 1993. The Spectral Image Processing System (SIPS) - Interactive Visualization and Analysis of Imaging Spectrometer Data. Remote Sensing of Environment 44, pp. 145-163.

Shen, H., Li, X., Cheng, Q., Zeng, C., Yang, G., Li, H. and Zhang, L., 2015. Missing information reconstruction of remote sensing 
data: A technical review. Geoscience and Remote Sensing Magazine, IEEE 3(3), pp. 61-85.

Tang, W., Shi, Z. and Wu, Y., 2014. Regularized simultaneous forward-backward greedy algorithm for sparse unmixing of hyperspectral data. Geoscience and Remote Sensing, IEEE Transactions on 52(9), pp. 5271-5288. 